| ISSN(online): 2589-8698 || ISSN(print): 2589-868X ||

\title{
LIPID PROFILE IN COPD PATIENTS
}

\section{Dr Ramavatar Bairwa ${ }^{1}$, Dr Pratibha Pareek ${ }^{2}$}

${ }^{1}$ Junior specialist, General medicine, RVRS Medical College and Attached MG Hospital Bhilwara Rajasthan.

${ }^{2}$ Associate Professor Zoology at MLV Govt. College Bhilwara

Article Info: Received 02 October 2021; Accepted 04 November 2021

DOI: https://doi.org/10.32553/ijmbs.v5i11.2291

Corresponding author: Dr Pratibha Pareek

Conflict of interest: No conflict of interest.

\section{Abstract}

Background: The COPD patients are expected to have a deranged lipid profile for various reasons like smoking, aging, and using such drugs as steroids. Aims of study to estimate the lipid profile level in COPD patients.

Methods: This cross-sectional study was conducted on 50 COPD patients and including 50 control were enrolled in the study. Results: The lipid profile in COPD patients, unpaired $t$ test was performed and it was observed that COPD patients showed significantly higher serum levels of TC, TG, LDL and serum concentrations of HDL were also decreased significantly compared to controls.

Conclusion: COPD patients showed significantly higher serum cholesterol level in COPD patients as compared to controls.

Keywords: Total cholesterol, TG, COPD.

\section{Introduction:}

Chronic obstructive pulmonary disease (COPD) is a common, preventable and treatable disease that is characterized by persistent respiratory symptoms and airflow limitation that is due to airway and/ or alveolar abnormalities usually caused by significant exposure to noxious particles or gases ${ }^{1}$.

Chronic obstructive pulmonary disease (COPD) is a multisystem condition defined as "a preventable and treatable disease with some significant extra-pulmonary effects that may contribute to the severity in individual patients." Its pulmonary component is characterized by airflow obstruction that is usually progressive and not fully reversible. In the western world, COPD is typically caused by long-term exposure to tobacco smoke ${ }^{2}$.

According to the statistics, chronic obstructive pulmonary disease (COPD) accounts for almost three million (5\%) deceases worldwide in 2015. This disease is likely to increase in the coming years due to the growing prevalence of smoking and population aging in many countries. Metabolic syndrome which includes central obesity, diabetes, hypertension, and hyperlipidaemia, is known entity in COPD patients. Metabolic syndrome was associated with markers of systemic inflammation like Interleukin-6, C reactive protein, and fibrinogen etc $^{3}$. There are limited number of studies investigating dyslipidemia in the COPD patients.

These studies have generally relied on questionnaires or diagnostic codes to determine the frequency of dyslipidemia in the patients with COPD, and it is unknown if dyslipidemia is another independent factor that could explain the increased risk of cardiovascular morbidity and mortality in the COPD patients ${ }^{4}$. The COPD patients are expected to have a deranged lipid profile for various reasons like smoking, aging, and using such drugs as steroids.

\section{Materials and Methods}

This cross-sectional study was conducted on the inpatients and outpatients attending the Department of General medicine, RVRS medical college and attached MG hospital Bhilwara Rajasthan. Therefore, a total of 50 COPD patients and including 50 controls were enrolled in the study.

\section{Inclusion Criteria}

Diagnosed cases of COPD

\section{Exclusion Criteria}

Uncooperativeness and unwillingness to participate in the study, Known case of carcinoma, bronchial asthma, active tuberculosis, diabetes, hypertension, collagen vascular disease, inability to properly perform spirometry, the presence of congenital or valvular cardiomyopathy or other familial hyperlipidemias.

After the informed consent was obtained from all the participants. Subsequently, the COPD patients were segregated and diagnosed based on the GOLD guidelines with such risk factors as dyspnea, chronic cough, chronic sputum production, history of exposure to the respective risk factors, and age of $>40$ years. a detailed history was obtained from all the patients; furthermore, clinical examination and radiological tests were performed to 
confirm the presence of COPD and the associated cardiovascular complications.

After a 12-hour overnight fast, $5 \mathrm{ml}$ fasting blood samples were collected from all the participants in the morning. The total cholesterol, HDL, and triglycerides were directly analyzed using the standard enzymatic techniques.

\section{Results}

Mean age in COPD patients was $36.19 \pm 16.35$ years and control patients was $36.50 \pm 15.30$ years. Both groups were well matched for age and sex distribution.

Table 1: Comparison of biochemical parameters in case and controls.

\begin{tabular}{|l|l|l|l|}
\hline Parameters & Case $(\mathrm{n}=50)$ & Control $(\mathrm{n}=50)$ & $\mathrm{p}$-value \\
\hline Mean Total cholesterol & $182.60 \pm 18.40$ & $171.20 \pm 14.40$ & $<0.05$ \\
\hline Mean LDL & $116.20 \pm 11.40$ & $108.50 \pm 11.20$ & $<0.05$ \\
\hline Mean HDL & $34.20 \pm 3.90$ & $36.30 \pm 4.80$ & $<0.05$ \\
\hline Mean Triglycerides & $142.90 \pm 11.60$ & $132.50 \pm 10.50$ & $<0.05$ \\
\hline
\end{tabular}

The lipid profile in COPD patients, unpaired t test was performed and it was observed that COPD patients showed significantly higher serum levels of TC, TG, LDL and serum concentrations of HDL were also decreased significantly compared to controls.

\section{Discussion}

COPD is associated with significantly increased morbidity and mortality and COPD precipitates dyslipidemia. So present was conducted and found that COPD patients showed significantly higher serum levels of TC, TG, LDL and serum concentrations of HDL were decreased significantly. This finding was corroborated with the results of some previous studies 4,5

Although Kamat SR, et al. In their study has shown the serum of lipid parameters are not different in COPD from healthy controls ${ }^{6}$. Smoking can cause major changes in serum lipid profile simultaneously smoking is a major risk factor in COPD. Smoking affects the lipid profile such a way that the plasma LDL, cholesterol and triglycerides concentration are higher and HDL cholesterol is lower in smoker than in nonsmokers ${ }^{7}$.

Nicotine causes the release of adrenaline from the adrenal cortex leading to increased serum concentration of free fatty acids (FFA) which stimulates hepatic synthesis and secretion of cholesterol as well as hepatic secretion of VLDL and hence increased TG. Smoking decreases estrogen levels and further leads to decreased HDL cholesterol concentration. Smoking also increases insulin resistance and LDL, VLDL and TG are elevated in this hyperinsulinemic conditions due to decreased activity of lipoprotein lipase ${ }^{8}$.

\section{Conclusion}

COPD patients showed significantly higher serum cholesterol level in COPD patients as compared to controls.

\section{References}

1. Murray \& Nadel's textbook of respiratory medicine sixth edition volume 1 [751].

2. Mohamed H. Zidan, Abdelmonem K. Rabie , Mohamed M. Megahed, Mahmoud Y. AbdelKhaleq. The usefulness of the DECAF score in predicting hospital mortality in Acute Exacerbations of Chronic Obstructive Pulmonary Disease. Egyptian Journal of Chest Diseases and Tuberculosis (2015) 64, 75-80.

3. Watz H, Waschki B, Kirsten A, Muller KC, Kretschmar G, Meyer T, et al. The metabolic syndrome in patients with chronic bronchitis and COPD: frequency and associated consequences for systemic inflammation and physical inactivity. Chest. 2009; 136:1039-46.

4. Chatila WM, Thomashow BM, Minai OA, Criner GJ, Make BJ. Comorbidities in chronic obstructive pulmonary disease. Proc Am Thorac Soc. 2008; 4:549-55.

5. Begum K, Begum MK, Sarker ZH, Dewan MRK, Siddique MJH. Lipid profile status of chronic obstructive pulmonary disease in hospitalized patients. Bangladesh $\mathrm{J}$ Med Biochem. 2010;3(2):42-45.

6. Nillawar AN, Joshi KB, Patil SB, Bardapurkar JS, Bardapurkar SJ. Evaluation of HS-CRP and Lipid Profile in COPD. J Clin Diagn Res. 2013;7:801-3.

7. Ebrahimi M, Kazemi-Bajestani SM, GhayourMobarhan M, Moohebati M, Paydar R, AzimiNezhad M, et al. Metabolic syndrome may not be a good predictor of coronary artery disease in the Iranian population: Population-specific definitions are required. Scientific World Journal. 2009;9:8696.

8. Rao MV, Raghu S, Kiran S, Rao CH. A study of lipid profile in chronic obstructive pulmonary disease. $\mathrm{J}$ of Evolution of Med and Dent Sci. 2015;4(42):7286-7295. DOI: $10.14260 /$ jemds /2015/1059. 